Analele Universităţii de Vest, Timişoara

Seria Matematică - Informatică

LIV , 1, (2016), 55- 71

\title{
Uniqueness of a Polynomial and a Differential Monomial Sharing a Small Function
}

\author{
Abhijit Banerjee and Molla Basir Ahamed
}

\begin{abstract}
In this paper taking a question in [1] into background we investigate the uniqueness of a non-constant polynomial with the differential monomial generated by a non-constant mermorphic function $f$. Our result will also extend a result of BanerjeeMajumder [2] given earlier. An open question is also posed, in the paper, for future investigation.
\end{abstract}

AMS Subject Classification (2000). 30D35

Keywords. Meromorphic function, uniqueness, differential monomial, small function

\section{A first section}

Let $f$ be a non-constant meromorphic function defined in the open complex plane $\mathbb{C}$. We adopt the standard notations of the Nevanlinna theory of meromorphic functions as explained in [4].

If for some $a \in \mathbb{C} \cup\{\infty\}, f$ and $g$ have the same set of $a$-points with the same multiplicities, we say that $f$ and $g$ share the value $a \mathrm{CM}$ (counting multiplicities) and if we do not consider the multiplicities then $f, g$ are said to share the value $a$ IM (ignoring multiplicities). When $a=\infty$ the zeros of $f-a$ means the poles of $f$.

It will be convenient to let $J$ denote any set of positive real numbers of finite linear measure, not necessarily the same at each occurrence. For any 
non-constant meromorphic function $f$, we denote by $S(r, f)$ any quantity satisfying

$$
S(r, f)=o(T(r, f)) \quad(r \longrightarrow \infty, r \notin J) .
$$

A meromorphic function $a=a(z)(\not \equiv \infty)$ is called a small function with respect to $f$ provided that $T(r, a)=S(r, f)$ as $r \longrightarrow \infty, r \notin J$. If $a=a(z)$ is a small function we define that $f$ and $g$ share $a$ IM or $a \mathrm{CM}$ according as $f-a$ and $g-a$ share 0 IM or 0 CM respectively. Also it is known to us that the hyper order of $f$, denoted by $\rho_{2}(f)$, is defined by

$$
\rho_{2}(f)=\limsup _{r \longrightarrow \infty} \frac{\log \log T(r, f)}{\log r} .
$$

We start our discussion on a well known result of Rubel and Yang ([10]), where they proved that if a non-constant entire function $f$ and $f^{\prime}$ share two distinct finite numbers $a, b \mathrm{CM}$, then $f=f^{\prime}$. This result is the starting point of the investigations about the relation between an entire or meromorphic function sharing some values with their derivatives.

In 1979, Mues and Steinmetz ([9]) obtained an analogous result for IM sharing. In this direction, in 1996, Brück ([3]) proposed his following famous conjecture.

Conjecture : Let $f$ be a non-constant entire function such that the hyper order $\rho_{2}(f)$ of $f$ is not a positive integer or infinite. If $f$ and $f^{\prime}$ share a finite value a $C M$, then $\frac{f^{\prime}-a}{f-a}=c$, where $c$ is a non-zero constant.

Brück himself proved the conjecture for $a=0$. For $a \neq 0$, Brück ([3]) obtained the following result in which additional supposition was required.

Theorem A. ([3]) Let $f$ be a non-constant entire function. If $f$ and $f^{\prime}$ share the value $1 C M$ and if $N\left(r, 0 ; f^{\prime}\right)=S(r, f)$ then $\frac{f^{\prime}-1}{f-1}$ is a nonzero constant.

Next we recall the following definitions.

Definition 1.1. ([12]) For $a \in \mathbb{C} \cup\{\infty\}$ and a positive integer $p$ we denote by

$$
N_{p}(r, a ; f)=\bar{N}(r, a ; f)+\bar{N}(r, a ; f \mid \geq 2)+\ldots+\bar{N}(r, a ; f \mid \geq p) .
$$

Clearly $N_{1}(r, a ; f)=\bar{N}(r, a ; f)$.

Definition 1.2. ([12]) For $a \in \mathbb{C} \cup\{\infty\}$ and a positive integer $p$ we put

$$
\delta_{p}(a, f)=1-\limsup _{r \rightarrow \infty} \frac{N_{p}(r, a ; f)}{T(r, f)} .
$$




\section{Clearly}

$0 \leq \delta(a, f) \leq \delta_{p}(a, f) \leq \delta_{p-1}(a, f) \leq \ldots \leq \delta_{2}(a, f) \leq \delta_{1}(a, f)=\Theta(a, f) \leq 1$

Definition 1.3. ([1]) For two positive integers $n, p$ we define $\mu_{p}=\min \{n, p\}$ and $\mu_{p}^{*}=p+1-\mu_{p}$. Then it is clear that

$$
N_{p}\left(r, 0 ; f^{n}\right) \leq \mu_{p} N_{\mu_{p}^{*}}(r, 0 ; f) .
$$

Definition 1.4. ([2]) Let $z_{0}$ be a zero of $f-a$ of multiplicity $p$ and a zero of $g-a$ of multiplicity $q$. We denote by $\bar{N}_{L}(r, a ; f)$ the counting function of those a-points of $f$ and $g$ where $p>q \geq 1$, by $N_{E}^{1)}(r, a ; f)$ the counting function of those a-points of $f$ and $g$ where $p=q=1$ and by $\bar{N}_{E}^{(2}(r, a ; f)$ the counting function of those a-points of $f$ and $g$ where $p=q \geq 2$, each point in these counting functions is counted only once. In the same way we can define $\bar{N}_{L}(r, a ; g), N_{E}^{1)}(r, a ; g), \bar{N}_{E}^{(2}(r, a ; g)$.

Definition 1.5. ([5]) Let $k$ be a nonnegative integer or infinity. For $a \in$ $\mathbb{C} \cup\{\infty\}$ we denote by $E_{k}(a ; f)$ the set of all a-points of $f$, where an a-point of multiplicity $m$ is counted $m$ times if $m \leq k$ and $k+1$ times if $m>k$. If $E_{k}(a ; f)=E_{k}(a ; g)$, we say that $f, g$ share the value a with weight $k$.

The definition implies that if $f, g$ share a value $a$ with weight $k$ then $z_{0}$ is an $a$-point of $f$ with multiplicity $m(\leq k)$ if and only if it is an $a$-point of $g$ with multiplicity $m(\leq k)$ and $z_{0}$ is an $a$-point of $f$ with multiplicity $m(>k)$ if and only if it is an $a$-point of $g$ with multiplicity $n(>k)$, where $m$ is not necessarily equal to $n$.

We write $f, g$ share $(a, k)$ to mean that $f, g$ share the value $a$ with weight $k$. Clearly if $f, g$ share $(a, k)$, then $f, g$ share $(a, p)$ for any integer $p$, $0 \leq p<k$. Also we note that $f, g$ share a value $a$ IM or CM if and only if $f, g$ share $(a, 0)$ or $(a, \infty)$ respectively.

With the notion of weighted sharing of values Lahiri-Sarkar ([6]) improved the result of Zhang ([11]). In ([12]) Zhang extended the result of Lahiri-Sarkar ([6]) and replaced the concept of value sharing by small function sharing.

In 2008, Zhang and Lü ([13]) obtained the following result.

Theorem B. ([13]) Let $k(\geq 1), n(\geq 1)$ be integers and $f$ be a non-constant meromorphic function. Also let $a(z)(\not \equiv 0, \infty)$ be a small function with respect to $f$. Suppose $f^{n}-a$ and $f^{(k)}-a$ share $(0, l)$. If $l=\infty$ and

$$
(3+k) \Theta(\infty, f)+2 \Theta(0, f)+\delta_{2+k}(0, f)>6+k-n
$$


or $l=0$ and

$$
(6+2 k) \Theta(\infty, f)+4 \Theta(0, f)+2 \delta_{2+k}(0, f)>12+2 k-n
$$

then $f^{n} \equiv f^{(k)}$.

In ([13]), Zhang and $L \ddot{u}$ asked this question :

Question 1.1. What will happen if $f^{n}$ and $\left[f^{(k)}\right]^{m}$ share a small function?

In 2010 Banerjee and Majumder ([2]) answer the above open question affirmatively in the following manner.

Theorem C. ([2]) Let $k(\geq 1), n(\geq 1), m(\geq 2)$ be integers and $f$ be a nonconstant meromorphic function. Also let $a(z)(\not \equiv 0, \infty)$ be a small function with respect to $f$. Suppose $f^{n}-a$ and $\left[f^{(k)}\right]^{m}-a$ share $(0, l)$. If $l=2$ and

$$
(3+2 k) \Theta(\infty, f)+2 \Theta(0, f)+2 \delta_{1+k}(0, f)>7+2 k-n
$$

or $l=1$ and

$$
\left(\frac{7}{2}+2 k\right) \Theta(\infty, f)+\frac{5}{2} \Theta(0, f)+2 \delta_{1+k}(0, f)>8+2 k-n
$$

or $l=0$ and

$$
(6+3 k) \Theta(\infty, f)+4 \Theta(0, f)+3 \delta_{1+k}(0, f)>13+3 k-n
$$

then $f^{n} \equiv\left[f^{(k)}\right]^{m}$.

Here we observe that in the conditions (1.3)-(1.5) there was no influence of $m$.

Next we recall the following definition.

Definition 1.6. ([4]) Let $n_{0 j}, n_{1 j}, \ldots, n_{k j}$ be nonnegative integers.

The expression $M_{j}[f]=(f)^{n_{0 j}}\left(f^{(1)}\right)^{n_{1 j}} \ldots\left(f^{(k)}\right)^{n_{k j}}$ is called a differential monomial generated by $f$ of degree $d_{M_{j}}=d\left(M_{j}\right)=\sum_{i=0}^{k} n_{i j}$ and weight $\Gamma_{M_{j}}=$ $\sum_{i=0}^{k}(i+1) n_{i j}$

The sum $P[f]=\sum_{j=1}^{t} b_{j} M_{j}[f]$ is called a differential polynomial generated by $f$ of degree $\bar{d}(P)=\max \left\{d\left(M_{j}\right): 1 \leq j \leq t\right\}$ and weight $\Gamma_{P}=\max \left\{\Gamma_{M_{j}}\right.$ : $1 \leq j \leq t\}$, where $T\left(r, b_{j}\right)=S(r, f)$ for $j=1,2, \ldots, t$. 
The numbers $\underline{d}(P)=\min \left\{d\left(M_{j}\right): 1 \leq j \leq t\right\}$ and $k$ (the highest order of the derivative of $f$ in $P[f]$ ) are called respectively the lower degree and order of $P[f]$.

$P[f]$ is said to be homogeneous if $\bar{d}(P)=\underline{d}(P)$.

$P[f]$ is called a linear differential polynomial generated by $f$ if $\bar{d}(P)=1$. Otherwise $P[f]$ is called a non-linear differential polynomial.

We denote by $Q=\max \left\{\Gamma_{M_{j}}-d\left(M_{j}\right): 1 \leq j \leq t\right\}=\max \left\{n_{1 j}+2 n_{2 j}+\right.$ $\left.\ldots+k n_{k j}: 1 \leq j \leq t\right\}$.

Also for the sake of convenience for a differential monomial $M[f]$ we denote by $\lambda=\Gamma_{M}-d_{M}$.

Since the natural extension of $\left[f^{(k)}\right]^{m}$ is a differential monomial, it will be interesting to see whether Theorem $C$ can remain true when $\left[f^{(k)}\right]^{m}$ is replaced by $M[f]$. In this direction, very recently Banerjee - Chakraborty ([1]) have improved Theorem $C$ in the following way which in turn improve a recent result of Li-Huang [7] as well.

Theorem D. ([1]) Let $k(\geq 1), n(\geq 1)$ be integers and $f$ be a non-constant meromorphic function and $M[f]$ be a differential monomial of degree $d_{M}$ and weight $\Gamma_{M}$ and $k$ is the highest derivative in $M[f]$. Also $a(z)(\not \equiv 0, \infty)$ be a small function with respect to $f$. Suppose $f^{n}-a$ and $M[f]-a$ share $(0, l)$. If $l \geq 2$ and

$$
(3+\lambda) \Theta(\infty, f)+\mu_{2} \delta_{\mu_{2}^{*}}(0, f)+d_{M} \delta_{k+2}(0 ; f)>\Gamma_{M}+3+\mu_{2}-n
$$

or $l=1$ and

$$
\left(\frac{7}{2}+\lambda\right) \Theta(\infty, f)+\frac{1}{2} \Theta(0 ; f)+\mu_{2} \delta_{\mu_{2}^{*}}(0, f)+d_{M} \delta_{k+2}(0 ; f)>\Gamma_{M}+4+\mu_{2}-n
$$

or $l=0$ and

$$
\begin{aligned}
& (6+2 \lambda) \Theta(\infty, f)+2 \Theta(0 ; f)+\mu_{2} \delta_{\mu_{2}^{*}}(0, f)+d_{M} \delta_{k+2}(0 ; f)+d_{M} \delta_{k+1}(0 ; f) \\
& >2 \Gamma_{M}+8+\mu_{2}-n
\end{aligned}
$$

then $f^{n} \equiv M[f]$.

In [1, Example 1.13], Banerjee-Chakraborty ([1]) have shown that $f^{n}$ can't be replaced by an arbitrary polynomial $P[f]=a_{0} f^{n}+a_{1} f^{n-1}+\ldots+a_{n}$ in Theorem $D$ for IM sharing case.

Observing Example 1.13 in [1] we note that $f(z)=e^{z}, P(f)=f^{2}+2 f$ and $M[f]=f^{(3)}$. So $P+1=(M+1)^{2}$. Thus $P$ and $M$ share $(-1,0)$. Also 
$\Theta(\infty ; f)=1=\Theta(0 ; f)=\delta_{q}(0 ; f)$. Here $p=n=1, m=1, w_{p}=0, \mu_{2}=1$, $d_{M}=1, \Gamma_{M}=4, \lambda=3$. Here

$$
\begin{aligned}
& (6+2 \lambda) \Theta(\infty, f)+2 \Theta(0 ; f)+\mu_{2} \delta_{\mu_{2}^{*}}(0, f)+d_{M} \delta_{k+2}(0 ; f)+d_{M} \delta_{k+1}(0 ; f) \\
& =17>16=2 \Gamma_{M}+8+\mu_{2}-n
\end{aligned}
$$

so that (1.8) is satisfied but $M[f] \not \equiv P[f]$. On the basis of this observation in $([1])$ following question was asked in [1]

Question 1.2. Is it possible to replace $f^{n}$ by arbitrary polynomial $P[f]=$ $a_{0} f^{n}+a_{1} f^{n-1}+\ldots+a_{n}$ in Theorem $D$ for $l \geq 1$ ?

In this paper, we will not only try to find the possible answer of the above Question 1.2, but also improve Theorem $D$ to a large extent. We have observed that if we consider the general polynomial $P(f)=a_{n} f^{n}+\ldots+a_{0}$ in the place of $f^{n}$ in the line of the proof of Theorem $D$, we will get a different inequality in comparison to (1.8) such that Example 1.13 is not violating the new condition (1.14) given later on.

Through the paper we shall assume the following notations. Let

$$
\mathcal{P}(w)=a_{n+m} w^{n+m}+\ldots+a_{n} w^{n}+\ldots+a_{0}=a_{n+m} \prod_{i=1}^{s}\left(w-w_{p_{i}}\right)^{p_{i}}
$$

where $a_{j}(j=0,1,2, \ldots, n+m-1), a_{n+m} \neq 0$ and $w_{p_{i}}(i=1,2, \ldots, s)$ are distinct finite complex numbers and $2 \leq s \leq n+m$ and $p_{1}, p_{2}, \ldots, p_{s}$, $s \geq 2, n, m$ and $k$ are all positive integers with $\sum_{i=1}^{s} p_{i}=n+m$. Also let $p>\max _{p \neq p_{i}, i=1, \ldots, r}\left\{p_{i}\right\}, r=s-1$, where $s$ and $r$ are two positive integers.

Let $P\left(w_{1}\right)=a_{n+m} \prod_{i=1}^{s-1}\left(w_{1}+w_{p}-w_{p_{i}}\right)^{p_{i}}=b_{q} w_{1}^{q}+b_{q-1} w_{1}^{q-1}+\ldots+b_{0}$, where $a_{n+m}=b_{q}, w_{1}=w-w_{p}, q=n+m-p$. Therefore, $\mathcal{P}(w)=w_{1}^{p} P\left(w_{1}\right)$.

Next we assume $P\left(w_{1}\right)=b_{q} \prod_{i=1}^{r}\left(w_{1}-\alpha_{i}\right)^{p_{i}}$, where $\alpha_{i}=w_{p_{i}}-w_{p}, \quad(i=$ $1,2 \ldots, r)$, be distinct zeros of $P\left(w_{1}\right)$.

The following theorem is the main result of this paper which gives an affirmative answer of the questions of Banerjee - Chakraborty ([1]) and also the question posed by Zhang-Lü ([13]) in a more convenient way.

Theorem 1.1. Let $k(\geq 1), n(\geq 1), p(\geq 1)$ and $m(\geq 0)$ be integers and $f$ and $f_{1}=f-w_{p}$ be two non-constant meromorphic functions and $M[f]$ be a differential monomial of degree $d_{M}$ and weight $\Gamma_{M}$ and $k$ is the highest 
derivative in $M[f]$. Let $\mathcal{P}(z)=a_{m+n} z^{m+n}+\ldots+a_{n} z^{n}+\ldots+a_{0}, a_{m+n} \neq 0$, be a polynomial in $z$ of degree $m+n$ such that $\mathcal{P}(f)=f_{1}^{p} P\left(f_{1}\right)$. Also let $a(z)(\not \equiv 0, \infty)$ be a small function with respect to $f$. Suppose $\mathcal{P}(f)-a$ and $M[f]-a$ share $(0, l)$. If $l \geq 2$ and

$$
(3+\lambda) \Theta(\infty, f)+\mu_{2} \delta_{\mu_{2}^{*}}\left(w_{p}, f\right)+d_{M} \delta_{2+k}(0, f)>\Gamma_{M}+\mu_{2}+3-p
$$

or $l=1$ and

$$
\begin{aligned}
& \left(\frac{7}{2}+\lambda\right) \Theta(\infty, f)+\frac{1}{2} \Theta\left(w_{p}, f\right)+\mu_{2} \delta_{\mu_{2}^{*}}\left(w_{p}, f\right)+d_{M} \delta_{2+k}(0, f) \\
> & \Gamma_{M}+\mu_{2}+4+\frac{(m+n)-3 p}{2}
\end{aligned}
$$

or $l=0$ and

$$
\begin{aligned}
& (6+2 \lambda) \Theta(\infty, f)+2 \Theta\left(w_{p}, f\right)+\mu_{2} \delta_{\mu_{2}^{*}}\left(w_{p}, f\right)+d_{M}\left(\sum_{i=1}^{2} \delta_{k+i}(0, f)\right) \\
> & 2 \Gamma_{M}+\mu_{2}+8+2(m+n)-3 p
\end{aligned}
$$

then $\mathcal{P}(f) \equiv M[f]$.

The following Corollary can easily be deduced from the above theorem which is an extension and improvement of the Theorem D. It is clear that for $P(z)=1$ i.e., $m=0$, we get exactly Theorem $\mathrm{E}$ from Corollary 1.1.

Corollary 1.1. Let $k(\geq 1), n(\geq 1)$ and $m(\geq 0)$ be integers and $f$ be a non-constant meromorphic function and $M[f]$ be a differential monomial of degree $d_{M}$ and weight $\Gamma_{M}$ and $k$ is the highest derivative in $M[f]$. Let $P(z)=$ $a_{m} z^{m}+\ldots+a_{0}, a_{m} \neq 0$, be a polynomial in $z$ of degree $m$. Also let $a(z)(\equiv$ $0, \infty)$ be a small function with respect to $f$. Suppose $f^{n} P(f)-a$ and $M[f]-a$ share $(0, l)$. If $l \geq 2$ and

$$
(3+\lambda) \Theta(\infty, f)+\mu_{2} \delta_{\mu_{2}^{*}}\left(w_{p}, f\right)+d_{M} \delta_{2+k}(0, f)>\Gamma_{M}+\mu_{2}+3-n
$$

or $l=1$ and

$$
\begin{aligned}
& \left(\frac{7}{2}+\lambda\right) \Theta(\infty, f)+\frac{1}{2} \Theta\left(w_{p}, f\right)+\mu_{2} \delta_{\mu_{2}^{*}}\left(w_{p}, f\right)+d_{M} \delta_{2+k}(0, f) \\
> & \Gamma_{M}+\mu_{2}+4+\frac{m}{2}-n
\end{aligned}
$$

or $l=0$ and

$$
\begin{aligned}
& (6+2 \lambda) \Theta(\infty, f)+2 \Theta\left(w_{p}, f\right)+\mu_{2} \delta_{\mu_{2}^{*}}\left(w_{p}, f\right)+d_{M}\left(\sum_{i=1}^{2} \delta_{k+i}(0, f)\right) \\
> & 2 \Gamma_{M}+\mu_{2}+8+2 m-n
\end{aligned}
$$

then $f^{n} P(f) \equiv M[f]$. 
We see that in case of Example 1.13 in [1] we have $\Theta(\infty ; f)=1=$ $\Theta(0 ; f)=\delta_{q}(0 ; f), p=n=1, m=1, w_{p}=0, \mu_{2}=1, d_{M}=1, \Gamma_{M}=4$, $\lambda=3$. So when $l=0$, we get

$$
\begin{aligned}
& (6+2 \lambda) \Theta(\infty, f)+2 \Theta\left(w_{p}, f\right)+\mu_{2} \delta_{\mu_{2}^{*}}\left(w_{p}, f\right)+d_{M}\left(\sum_{i=1}^{2} \delta_{k+i}(0, f)\right) \\
= & 17<18=2 \Gamma_{M}+\mu_{2}+8+2 m-n,
\end{aligned}
$$

thus (1.14) ceases to hold and hence Example 1.13 [1] is not violating Corollary 1.1.

However the following question is still open.

Question 1.3. Is it possible to extend Theorem 1.1 up to differential polynomial $P[f]$ instead of differential monomial $M[f]$ ?

Following example shows that in Theorem $1.1 a(z) \not \equiv 0$ is essential.

Example 1.1. Let us take $f(z)=e^{N z}$ where $N \neq 0, \pm 1$ and $\mathcal{P}(f)=f^{3}$, $M[f]=f^{(2)}$. Then $\mathcal{P}(f)$ and $M[f]$ share $a=0$ (or, $\left.\infty\right)$. Here $m=0, p=n=$ $1, w_{p}=0, d_{M}=1, \mu_{2}=1, \Gamma_{M}=3$ and $\lambda=2$. Also $\Theta(\infty ; f)=1=\Theta(0 ; f)$ and $\delta_{q}(0 ; f)=1, \forall q \in \mathbb{N}$. Thus we see that the deficiency conditions stated in Theorem 1.1 are satisfied but $\mathcal{P}(f) \not \equiv M[f]$.

The next example shows that the deficiency conditions stated in Theorem 1.1 are not necessary.

Example 1.2. Let $f(z)=A \cos z+B \sin z, A B \neq 0$. Then $\bar{N}(r, f)=S(r, f)$ and

$$
\bar{N}(r, 0 ; f)=\bar{N}\left(r, \frac{A+i B}{A-i B} ; e^{2 i z}\right) \sim T(r, f) .
$$

Here $m=0, p=n=1, w_{p}=0, d_{M}=1, \mu_{2}=1, \Gamma_{M}=4 k+1$ and $\lambda=4 k$. Again $\Theta(\infty, f)=1$ and $\Theta(0, f)=\delta_{p}(0, f)=0$. Let $m=0$, hence $\mathcal{P}(f)=f$.

Therefore it is clear that $M[f]=f^{(4 k)}$, for $k \in \mathbb{N}$ and $\mathcal{P}(f)$ share $a(z)$ and the deficiency conditions in Theorem 1.1 are not satisfied, but $\mathcal{P}(f) \equiv M$.

The following three examples show that the conditions (1.9) - (1.11) in Theorem 1.1 can not be removed.

Example 1.3. Let $f(z)=e^{N z}$, where $N$ is a non-zero integer. For $n \geq 2$ let

$$
\mathcal{P}(f)=-N^{2 n} \sum_{r=0}^{2 n-1}(-1)^{r}\left(\begin{array}{c}
2 n \\
r
\end{array}\right) f^{2 n-r} \text { and } M[f]=f^{(2 n)} .
$$

Then it is clear that

$$
\mathcal{P}(f)-N^{2 n}=-N^{2 n}\left(e^{N z}-1\right)^{2 n} \text { and } M[f]-N^{2 n}=N^{2 n}\left(e^{N z}-1\right) .
$$


Thus we see that $\mathcal{P}(f)$ and $M[f]$ share $\left(N^{2 n}, 0\right)$. Here $n+m=2 n, p=1$, $w_{p}=0, d_{M}=1, \Gamma_{M}=2 n+1, \mu_{2}=1$ and $\lambda=2 n$. Also $\Theta(\infty ; f)=1=$ $\Theta(0 ; f)$ and $\delta_{q}(0 ; f)=1, \forall q \in \mathbb{N}$.

So for $l=0$

$$
\begin{aligned}
& (6+2 \lambda) \Theta(\infty, f)+2 \Theta\left(w_{p}, f\right)+\mu_{2} \delta_{\mu_{2}^{*}}\left(w_{p}, f\right)+d_{M}\left(\sum_{i=1}^{2} \delta_{k+i}(0, f)\right) \\
= & 4 n+11 \leq 8 n+8=2 \Gamma_{M}+\mu_{2}+8+2(m+n)-3 p
\end{aligned}
$$

and we see that $\mathcal{P}(f) \not \equiv M[f]$.

Example 1.4. Let $f(z)=-\sin (\alpha z)+a-\frac{a}{\alpha^{4 k}}, k \in \mathbb{N} ;$ where $\alpha \neq 0, \alpha^{4 k} \neq 1$ and $a \in \mathbb{C}-\{0\}$. Let $p=n=1, w_{p}=0$ and $m=0$. Then let $\mathcal{P}(f)=f$. Again let $M[f]=f^{(4 k)}$. Then $M[f]=-\alpha^{4 k} \sin (\alpha z)$. Here $m=0, \mu_{2}=$ $1, \Gamma_{M}=4 k+1, d_{M}=1$ and $\lambda=4 k$. Again $\Theta(\infty ; f)=1$ and

$$
\bar{N}(r, 0 ; f)=\bar{N}\left(r, a-\frac{a}{\alpha^{4 k}} ; \sin (\alpha z)\right) \sim T(r, f) .
$$

Therefore,

$$
\Theta(0 ; f)=0=\delta_{q}(0 ; f), \forall q \in \mathbb{N} .
$$

Also it is clear that $\mathcal{P}(f)$ and $M[f]$ share $(a, l)(l \geq 0)$ but none of the inequalities (1.9), (1.10) and (1.11) of Theorem 1.1 is satisfied and $\mathcal{P}(f) \not \equiv$ $M[f]$.

Example 1.5. Let $f(z)=e^{\beta z}+a-\frac{a}{\beta^{2}}$; where $a \neq 0, \infty$ and $\beta \neq 0, \pm 1$. Let $p=n=1, w_{p}=0$ and $m=0$. Then let $\mathcal{P}(f)=f$. Again let $M[f]=f^{(2)}$. Then $M[f]=\beta^{2} e^{\beta z}$. Here $m=0, \mu_{2}=1, \Gamma_{M}=3, d_{M}=1$ and $\lambda=2$. Again $\Theta(\infty ; f)=1$ and

$$
\bar{N}(r, 0 ; f)=\bar{N}\left(r, \frac{a}{\beta^{2}}-a ; e^{\beta z}\right) \sim T(r, f) .
$$

Therefore,

$$
\Theta(0 ; f)=0=\delta_{q}(0 ; f), \forall q \in \mathbb{N} .
$$

Also it is clear that $\mathcal{P}(f)$ and $M[f]$ share $(a, l)(l \geq 0)$ but none of the inequalities (1.9), (1.10) and (1.11) of Theorem 1.1 is satisfied and $\mathcal{P}(f) \not \equiv$ $M[f]$. 


\section{Lemmas}

In this section we present some Lemmas which will be needed in the sequel. Let $F, G$ be two non-constant meromorphic functions. Henceforth we shall denote by $H$ the following function.

$$
H=\left(\frac{F^{\prime \prime}}{F^{\prime}}-\frac{2 F^{\prime}}{F-1}\right)-\left(\frac{G^{\prime \prime}}{G^{\prime}}-\frac{2 G^{\prime}}{G-1}\right) .
$$

Lemma 2.1. [8] Let $f$ be a non-constant meromorphic function and let

$$
R(f)=\frac{\sum_{i=0}^{n} a_{i} f^{i}}{\sum_{j=0}^{m} b_{j} f^{j}}
$$

be an irreducible rational function in $f$ with constant coefficients $\left\{a_{i}\right\}$ and $\left\{b_{j}\right\}$ where $a_{n} \neq 0$ and $b_{m} \neq 0$. Then

$$
T(r, R(f))=d T(r, f)+S(r, f),
$$

where $d=\max \{n, m\}$.

Lemma 2.2. ([1]) For any non-constant meromorphic function $f$,

$$
N\left(r, \infty ; \frac{M}{f^{d_{M}}}\right) \leq d_{M} N(r, 0 ; f)+\lambda \bar{N}(r, \infty ; f)+S(r, f) .
$$

Lemma 2.3. ([1]) For the differential monomial $M[f]$,

$$
N_{p}(r, 0 ; M[f]) \leq d_{M} N_{p+k}(r, 0 ; f)+\lambda \bar{N}(r, \infty ; f)+S(r, f) .
$$

Lemma 2.4. Let $f$ be a non-constant meromorphic function and $a(z)$ be a small function in $f$. Let us define $F=\frac{\mathcal{P}(f)}{a}=\frac{f_{1}^{p} P\left(f_{1}\right)}{a}$ and $G=\frac{M[f]}{a}$. Then $F G \not \equiv 1$.

Proof. On contrary suppose $F G \equiv 1$ i.e

$$
f_{1}^{p} P\left(f_{1}\right) M[f]=a^{2} .
$$

From above it is clear that the function $f$ can't have any zeros and poles. Therefore

$$
\bar{N}(r, 0 ; f)=S(r, f)=\bar{N}(r, \infty ; f) .
$$


So by the First Fundamental Theorem and Lemma 2.1, we have

$$
\begin{aligned}
\left(m+n+d_{M}\right) T(r, f) & =T\left(r, \frac{a^{2}}{f_{1}^{p} P\left(f_{1}\right) f^{d_{M}}}\right)+S(r, f) \\
& \leq T\left(r, \frac{M[f]}{f^{d_{M}}}\right)+S(r, f) \\
& \leq m\left(r, \frac{M[f]}{f^{d_{M}}}\right)+N\left(r, \frac{M[f]}{f^{d_{M}}}\right)+S(r, f) \\
& \leq N\left(r, \frac{M[f]}{f^{d_{M}}}\right)+S(r, f) .
\end{aligned}
$$

Then using Lemma 2.2 and from above inequality, we get

$$
\left(m+n+d_{M}\right) T(r, f) \leq d_{M} N(r, 0 ; f)+\lambda \bar{N}(r, f)+S(r, f) \leq S(r, f),
$$

which is not possible.

Lemma 2.5. ([2]) Let $F$ and $G$ share $(1, l)$ and $\bar{N}(r, F)=\bar{N}(r, G)$ and $H \not \equiv 0$, where $F, G$ and $H$ are defined as earlier. Then

$$
\begin{aligned}
& N(r, \infty ; H) \\
\leq & \bar{N}(r, \infty ; F)+\bar{N}(r, 0 ; F \mid \geq 2)+\bar{N}(r, 0 ; G \mid \geq 2)+\bar{N}_{0}\left(r, 0 ; F^{\prime}\right) \\
& +\bar{N}_{0}\left(r, 0 ; G^{\prime}\right)+\bar{N}_{L}(r, 1 ; F)+\bar{N}_{L}(r, 1 ; G)+S(r, f) .
\end{aligned}
$$

Lemma 2.6. ([1]) Let $F$ and $G$ share $(1, l)$. Then

$$
\bar{N}_{L}(r, 1 ; F) \leq \frac{1}{l+1} \bar{N}(r, \infty ; F)+\frac{1}{l+1} \bar{N}(r, 0 ; F)+S(r, F) \quad \text { if } l \geq 1,
$$

and

$$
\bar{N}_{L}(r, 1 ; F) \leq \bar{N}(r, \infty ; F)+\bar{N}(r, 0 ; F)+S(r, F) \quad \text { if } l=0 .
$$

Lemma 2.7. ([1]) Let $F$ and $G$ share $(1, l)$ and $H \not \equiv 0$. Then

$$
\begin{aligned}
& \bar{N}(r, 1 ; F)+\bar{N}(r, 1 ; G) \\
\leq & N(r, \infty ; H)+\bar{N}_{E}^{(2}(r, 1 ; F)+\bar{N}_{L}(r, 1 ; F)+\bar{N}_{L}(r, 1 ; G) \\
& +\bar{N}(r, 1 ; G)+S(r, f) .
\end{aligned}
$$

The following lemma can be proved in the line of proof of Lemma 2.14 [1].

Lemma 2.8. Let $f$ be a non-constant meromorphic function and $a(z)$ be a small function of $f$. Let $F=\frac{\mathcal{P}(f)}{a}=\frac{f_{1}^{p} P\left(f_{1}\right)}{a}$ and $G=\frac{M[f]}{a}$ such that $F$ and $G$ shares $(1, \infty)$. Then one of the following cases holds: 
1. $T(r) \leq N_{2}(r, 0 ; F)+N_{2}(r, 0 ; G)+\bar{N}(r, \infty ; F)+\bar{N}(r, \infty ; G)+\bar{N}_{L}(r, \infty ; F)$ $+\bar{N}_{L}(r, \infty ; G)+S(r)$

2. $F \equiv G$,

3. $F G \equiv 1$.

where $T(r)=\max \{T(r, F), T(r, G)\}$ and $S(r)=o(T(r)), r \in I, I$ is a set of infinite linear measure of $r \in(0, \infty)$.

\section{Proof of the theorem}

Proof of Theorem 1.1. Let $F=\frac{\mathcal{P}(f)}{a}=\frac{f_{1}^{p} P\left(f_{1}\right)}{a}$ and $G=\frac{M[f]}{a}$. Then $F-1=\frac{f_{1}^{p} P\left(f_{1}\right)-a}{a}$ and $G-1=\frac{M[f]-a}{a}$. Since $\mathcal{P}(f)$ and $M[f]$ share $(a, l)$, it follows that $F$ and $G$ share $(1, l)$ except the zeros and poles of $a(z)$. Now we consider the following cases.

Case 1 Let $H \not \equiv 0$.

Subcase 1.1. Let $l \geq 1$.

Using the Second Fundamental Theorem and Lemmas 2.7, 2.5 we get

$$
\begin{aligned}
& T(r, F)+T(r, G) \\
\leq & \bar{N}(r, \infty ; F)+\bar{N}(r, \infty ; G)+\bar{N}(r, 0 ; F)+\bar{N}(r, 0 ; G)+\bar{N}(r, 1 ; F) \\
& +\bar{N}(r, 1 ; G)-\bar{N}_{0}\left(r, 0 ; F^{\prime}\right)-\bar{N}_{0}\left(r, 0 ; G^{\prime}\right)+S(r, F)+S(r, G) \\
\leq & \bar{N}(r, \infty ; F)+\bar{N}(r, \infty ; G)+\bar{N}(r, 0 ; F)+\bar{N}(r, 0 ; G)+N(r, H) \\
& +\bar{N}_{E}^{(2}(r, 1 ; F)+\bar{N}_{L}(r, 1 ; F)+\bar{N}_{L}(r, 1 ; G)+\bar{N}(r, 1 ; G) \\
& -\bar{N}_{0}\left(r, 0 ; F^{\prime}\right)-\bar{N}_{0}\left(r, 0 ; G^{\prime}\right)+S(r, f) \\
\leq & 2 \bar{N}(r, \infty ; F)+\bar{N}(r, \infty ; G)+N_{2}(r, 0 ; F)+N_{2}(r, 0 ; G)+\bar{N}_{E}^{(2}(r, 1 ; F) \\
& +2 \bar{N}_{L}(r, 1 ; F)+2 \bar{N}_{L}(r, 1 ; G)+\bar{N}(r, 1 ; G)+S(r, f) .
\end{aligned}
$$


Subsubcase 1.1.1. Suppose $l=1$.

Then from the above inequality and using Lemmas 2.6, 2.3 we get

$$
\begin{aligned}
& T(r, F)+T(r, G) \\
\leq & 2 \bar{N}(r, \infty ; F)+\bar{N}(r, \infty ; G)+N_{2}(r, 0 ; F)+N_{2}(r, 0 ; G)+\bar{N}_{E}^{(2}(r, 1 ; F) \\
& +2 \bar{N}_{L}(r, 1 ; F)+2 \bar{N}_{L}(r, 1 ; G)+\bar{N}(r, 1 ; G)+S(r, f) \\
\leq & 3 \bar{N}(r, \infty ; F)+N_{2}\left(r, 0 ; f_{1}^{p} P\left(f_{1}\right)\right)+N_{2}(r, 0 ; M[f])+\frac{1}{2} \bar{N}(r, \infty ; F) \\
& +\frac{1}{2} \bar{N}(r, 0 ; F)+N(r, 1 ; G)+S(r, f) \\
\leq & 3 \bar{N}(r, \infty ; f)+\mu_{2} N_{\mu_{2}^{*}}\left(r, w_{p} ; f\right)+(m+n-p) T(r, f)+d_{M} N_{k+2}(r, 0 ; f) \\
& +\lambda \bar{N}(r, \infty ; f)+\frac{1}{2} \bar{N}(r, \infty ; F)+\frac{1}{2} \bar{N}(r, 0 ; F)+N(r, 1 ; G)+S(r, f) \\
\leq & \left(\frac{7}{2}+\lambda\right) \bar{N}(r, \infty ; f)+\mu_{2} N_{\mu_{2}^{*}}\left(r, w_{p} ; f\right)+(m+n-p) T(r, f) \\
& +d_{M} N_{k+2}(r, 0 ; f)+\frac{1}{2} \bar{N}\left(r, w_{p} ; f\right)+\frac{1}{2}(m+n-p) T(r, f)+N(r, 1 ; G) \\
& +S(r, f) . \\
\leq & \left(\frac{7}{2}+\lambda\right) \bar{N}(r, \infty ; f)+\mu_{2} N_{\mu_{2}^{*}}\left(r, w_{p} ; f\right)+\frac{3}{2}(m+n-p) T(r, f) \\
& +d_{M} N_{k+2}(r, 0 ; f)+\frac{1}{2} \bar{N}\left(r, w_{p} ; f\right)+T(r, G)+S(r, f) .
\end{aligned}
$$

i.e., in view of Lemma 2.1, for any given $\varepsilon>0$

$$
\begin{aligned}
& (m+n) T(r, f) \\
\leq & \left(\frac{7}{2}+\lambda\right) \bar{N}(r, \infty ; f)+\mu_{2} N_{\mu_{2}^{*}}\left(r, w_{p} ; f\right)+\frac{3}{2}(m+n-p) T(r, f) \\
& +d_{M} N_{k+2}(r, 0 ; f)+\frac{1}{2} \bar{N}\left(r, w_{p} ; f\right)+S(r, f) . \\
\leq & \left\{\left(\frac{7}{2}+\lambda\right)-\left(\frac{7}{2}+\lambda\right) \Theta(\infty, f)+\frac{1}{2}-\frac{1}{2} \Theta\left(w_{p}, f\right)+\mu_{2}-\mu_{2} \delta_{\mu_{2}^{*}}\left(w_{p}, f\right)\right. \\
& \left.+d_{M}-d_{M} \delta_{2+k}(0, f)+\frac{3}{2}(m+n-p)+\varepsilon\right\} T(r, f)+S(r, f) .
\end{aligned}
$$

i.e.,

$$
\begin{aligned}
& \left\{\left(\frac{7}{2}+\lambda\right) \Theta(\infty, f)+\frac{1}{2} \Theta\left(w_{p}, f\right)+\mu_{2} \delta_{\mu_{2}^{*}}\left(w_{p}, f\right)+d_{M} \delta_{2+k}(0, f)-\varepsilon\right\} T(r, f) \\
\leq & \left(\Gamma_{M}+\mu_{2}+4+\frac{(m+n)-3 p}{2}\right) T(r, f)+S(r, f),
\end{aligned}
$$


which is a contradiction.

Subsubcase 1.1.2. Suppose $l \geq 2$.

Here by using Lemma 2.3, we obtained

$$
\begin{aligned}
& T(r, F)+T(r, G) \\
\leq & 2 \bar{N}(r, \infty ; F)+\bar{N}(r, \infty ; G)+N_{2}(r, 0 ; F)+N_{2}(r, 0 ; G)+\bar{N}_{E}^{(2}(r, 1 ; F) \\
& +2 \bar{N}_{L}(r, 1 ; F)+2 \bar{N}_{L}(r, 1 ; G)+\bar{N}(r, 1 ; G)+S(r, f) \\
\leq & 3 \bar{N}(r, \infty ; f)+\mu_{2} N_{\mu_{2}^{*}}\left(r, w_{p} ; f\right)+(m+n-p) T(r, f)+d_{M} N_{k+2}(r, 0 ; f) \\
& +\lambda \bar{N}(r, \infty ; f)+N(r, 1 ; G)+S(r, f) \\
\leq & (3+\lambda) \bar{N}(r, \infty ; f)+\mu_{2} N_{\mu_{2}^{*}}\left(r, w_{p} ; f\right)+(m+n-p) T(r, f) \\
+ & d_{M} N_{k+2}(r, 0 ; f)+T(r, G)+S(r, f) .
\end{aligned}
$$

So, in view of Lemma 2.1, for any given $\varepsilon>0$ we have

$$
\begin{aligned}
& (m+n) T(r, f) \\
\leq & (3+\lambda) \bar{N}(r, \infty ; f)+\mu_{2} N_{\mu_{2}^{*}}\left(r, w_{p} ; f\right)+(m+n-p) T(r, f) \\
+ & d_{M} N_{2+k}(r, 0 ; f)+S(r, f) \\
\leq & \left\{(3+\lambda)-(3+\lambda) \Theta(\infty, f)+\mu_{2}-\mu_{2} \delta_{\mu_{2}^{*}}\left(w_{p}, f\right)+d_{M}-d_{M} \delta_{2+k}(0, f)\right. \\
+ & (m+n-p)+\varepsilon\} T(r, f)+S(r, f)
\end{aligned}
$$

i.e.,

$$
\begin{aligned}
& \left\{(3+\lambda) \Theta(\infty, f)+\mu_{2} \delta_{\mu_{2}^{*}}\left(w_{p}, f\right)+d_{M} \delta_{2+k}(0, f)-\varepsilon\right\} T(r, f) \\
\leq & \left(\Gamma_{M}+\mu_{2}+3-p\right) T(r, f)+S(r, f),
\end{aligned}
$$

which is a contradiction.

Subcase 1.2. Suppose $l=0$.

Then in view of the Second Fundamental Theorem and Lemmas 2.7, 2.5 we get

$$
\begin{aligned}
& T(r, F)+T(r, G) \\
\leq & \bar{N}(r, \infty ; F)+\bar{N}(r, 0 ; F)+\bar{N}(r, 1 ; F)+\bar{N}(r, \infty ; G)+\bar{N}(r, 0 ; G) \\
& +\bar{N}(r, 1 ; G)-\bar{N}_{0}\left(r, 0 ; F^{\prime}\right)-\bar{N}_{0}\left(r, 0 ; G^{\prime}\right)+S(r, F)+S(r, G) \\
\leq & \bar{N}(r, \infty ; F)+\bar{N}(r, 0 ; F)+\bar{N}(r, \infty ; G)+\bar{N}(r, 0 ; G)+N(r, \infty ; H) \\
& +\bar{N}_{E}^{(2}(r, 1 ; F)+\bar{N}_{L}(r, 1 ; F)+\bar{N}_{L}(r, 1 ; G)+\bar{N}(r, 1 ; G) \\
& -\bar{N}_{0}\left(r, 0 ; F^{\prime}\right)-\bar{N}_{0}\left(r, 0 ; G^{\prime}\right)+S(r, F)+S(r, G) \\
\leq & 2 \bar{N}(r, \infty ; F)+\bar{N}(r, \infty ; G)+N_{2}(r, 0 ; F)+N_{2}(r, 0 ; G)+\bar{N}_{E}^{(2}(r, 1 ; F) \\
& +2 \bar{N}_{L}(r, 1 ; F)+2 \bar{N}_{L}(r, 1 ; G)+\bar{N}(r, 1 ; G)+S(r, f)
\end{aligned}
$$


Again by Lemmas 2.6, 2.3 we get from above

$$
\begin{aligned}
& T(r, F)+T(r, G) \\
\leq & 2 \bar{N}(r, \infty ; F)+\bar{N}(r, \infty ; G)+\mu_{2} N_{\mu_{2}^{*}}\left(r, w_{p}, f\right)+(m+n-p) T(r, f) \\
& +N_{2}(r, 0 ; G)+2(\bar{N}(r, \infty ; F)+\bar{N}(r, 0 ; F))+\bar{N}(r, \infty ; G)+\bar{N}(r, 0 ; G) \\
& +\bar{N}_{E}^{(2}(r, 1 ; F)+\bar{N}_{L}(r, 1 ; G)+\bar{N}(r, 1 ; G)+S(r, f) \\
\leq & 4 \bar{N}(r, \infty ; F)+\mu_{2} N_{\mu_{2}^{*}}\left(r, w_{p}, f\right)+(m+n-p) T(r, f)+N_{2}(r, 0 ; G) \\
& +2 \bar{N}(r, \infty ; G)+\bar{N}(r, 0 ; G)+2 \bar{N}(r, 0 ; F)+T(r, G)+S(r, f)
\end{aligned}
$$

i.e., for any given $\varepsilon>0$

$$
\begin{aligned}
& (m+n) T(r, f) \\
\leq & (6+2 \lambda) \bar{N}(r, \infty ; f)+2 \bar{N}\left(r, w_{p} ; f\right)+2(m+n-p) T(r, f) \\
+ & \mu_{2} N_{\mu_{2}^{*}}\left(r, w_{p}, f\right)+(m+n-p) T(r, f)+d_{M} N_{1+k}(r, 0 ; f) \\
+ & d_{M} N_{2+k}(r, 0 ; f)+S(r, f) \\
\leq & \left\{(6+2 \lambda)-(6+2 \lambda) \Theta(\infty, f)+2-2 \Theta\left(w_{p}, f\right)+\mu_{2}-\mu_{2} \delta_{\mu_{2}^{*}}\left(w_{p}, f\right)\right. \\
& \left.+2 d_{M}-d_{M}\left(\sum_{i=1}^{2} \delta_{k+i}(0, f)\right)+3(m+n-p)+\varepsilon\right\} T(r, f)+S(r, f) .
\end{aligned}
$$

i.e.,

$$
\begin{aligned}
& \left\{(6+2 \lambda) \Theta(\infty, f)+2 \Theta\left(w_{p}, f\right)+\mu_{2} \delta_{\mu_{2}^{*}}\left(w_{p}, f\right)\right. \\
& \left.+d_{M}\left(\sum_{i=1}^{2} \delta_{k+i}(0, f)\right)-\varepsilon\right\} T(r, f) \\
\leq & \left(2 \Gamma_{M}+\mu_{2}+8+2(m+n)-3 p\right) T(r, f)+S(r, f),
\end{aligned}
$$

which is a contradiction.

Case 2. Let $H \equiv 0$.

On Integration we get,

$$
\frac{1}{G-1} \equiv \frac{A}{F-1}+B
$$

where $A(\neq 0), B$ are complex constants.

It is clear that $F$ and $G$ share $(1, \infty)$. Also by construction of $F$ and $G$ we see that $F$ and $G$ share $(\infty, 0)$ also. 
So using Lemma 2.3 and condition (1.9), we obtain

$$
\begin{aligned}
& N_{2}(r, 0 ; F)+N_{2}(r, 0 ; G)+\bar{N}(r, \infty ; F)+\bar{N}(r, \infty ; G)+\bar{N}_{L}(r, \infty ; F) \\
+ & \bar{N}_{L}(r, \infty ; G)+S(r) \\
\leq & \mu_{2} N_{\mu_{2}^{*}}\left(r, w_{p} ; f\right)+N_{2}(r, 0 ; P(f))+d_{M} N_{2+k}(r, 0 ; f)+\lambda \bar{N}(r, \infty ; f) \\
+ & 3 \bar{N}(r, \infty ; f)+S(r) \\
\leq & \mu_{2} N_{\mu_{2}^{*}}\left(r, w_{p} ; f\right)+(m+n-p) T(r, f)+d_{M} N_{2+k}(r, 0 ; f) \\
+ & (3+\lambda) \bar{N}(r, \infty ; f)+S(r) \\
\leq & \left\{\left(3+\lambda+d_{M}+\mu_{2}+m+n-p\right)-\left((\lambda+3) \Theta(\infty, f)+\mu_{2} \delta_{\mu_{2}^{*}}\left(w_{p}, f\right)\right.\right. \\
+ & \left.d_{M} \delta_{2+k}(0, f)\right) \\
& T(r, f)+S(r) \\
\leq & \left\{\left(3+\Gamma_{M}+\mu_{2}+m+n-p\right)-\left(3+\Gamma_{M}+\mu_{2}-p\right)\right\} T(r, f)+S(r) \\
\leq & (m+n) T(r, f)+S(r) \\
< & T(r, F)+S(r) .
\end{aligned}
$$

Hence inequality (1) of Lemma 2.8 does not hold. Again in view of Lemma 2.4 , we get $F G \not \equiv 1$. Therefore $F \equiv G$ i.e, $\mathcal{P}(f) \equiv M[f]$.

\section{Acknowledgement}

This research work is supported by the Council Of Scientific and Industrial Research, Extramural Research Division, CSIR Complex, Pusa, New Delhi110012, India, under the sanction project no. 25(0229)/14/EMR-II.

\section{References}

[1] A. Banerjee and B. Chakraborty, Further investigations on a question of Zhang and Lü, Ann. Univ. Paedagog. Crac. Stud. Math., 14, (2015), 105-119.

[2] A. Banerjee and S. Majumder, On the uniqueness of a power of a meromorphic function sharing a small function with the power of its derivative, Comment. Math. Univ. Carolin., 51, (2010), 565-576.

[3] R. Brück, On entire functions which share one value CM with their first derivative, Results Math., 30, (1996), 21-24.

[4] W. K. Hayman, Meromorphic Functions, The Clarendon Press, Oxford, 1964

[5] I. Lahiri, Weighted value sharing and uniqueness of meromorphic functions, Complex Var. Theory Appl., 46, (2001), 241-253. 
[6] I. Lahiri and A. Sarkar, Uniqueness of meromorphic function and its derivative, J.Inequal.Pure Appl. Math., 5, (2004), 1-9.

[7] J. D. Li and G. X. Huang, On meromorphic functions that share one small function with their derivatives, Palestine J. Math., 4, (2015), 91-96.

[8] A. Z. Mokhon'ko, On the Nevanlinna characteristics of some meromorphic functions, Izd-vo Khar'kovsk, Un-ta, 14, (1971), 83-87.

[9] E. Mues and N. Steinmetz, Meromorphe Funktionen die unit ihrer Ableitung Werte teilen, Manuscripta Math., 29, (1979), 195-206.

[10] L. A. Rubel and C. C. Yang, Values shared by an entire function and its derivative, Complex analysis (Proc. Conf., Univ. Kentucky, Lexington, Ky., 1976), Lecture Notes in Math., 599, (1977), 101-103.

[11] Q. C. Zhang, The uniqueness of meromorphic functions with their derivatives, Kodai Math. J., 21, (1998), 170-184.

[12] Q. C. Zhang, Meromorphic function that shares one small function with its derivative, J. Inequal. Pure Appl. Math., 6, (2015), 1-13.

[13] T. D. Zhang and W. R. L $\ddot{u}$, Notes on meromorphic function sharing one small function with its derivative, Complex Var. Ellip. Eqn., 53, (2008), 857-867.

\author{
Abhijit Banerjee \\ Department of Mathematics, \\ University of Kalyani, \\ Nadia, \\ West Bengal, 741235 \\ India \\ E-mail: abanerjee_kal@yahoo.co.in, abanerjeekal@gmail.com \\ Molla Basir Ahamed \\ Department of Mathematics, \\ Kalipada Ghosh Tarai Mahavidyalaya, \\ Bagdogra, Darjeeling, \\ West Bengal, 734014, \\ India \\ E-mail: bsrhmd116@gmail.com, bsrhmd117@gmail.com
}

Received: 30.05 .2016

Accepted: 25.07.2016 